\title{
The Hard Quiescent Spectrum of the Neutron Star X-ray Transient Exo 1745-248 in the Globular Cluster Terzan 5
}

\section{Citation}

Wijnands, Rudy, Craig 0. Heinke, David Pooley, Peter D. Edmonds, Walter H. G. Lewin, Jonathan E. Grindlay, Peter G. Jonker, and Jon M. Miller. 2005. "The Hard Quiescent Spectrum of the Neutron Star X\#Ray Transient EXO 1745-248 in the Globular Cluster Terzan 5." The Astrophysical Journal 618 (2): 883-90. https://doi.org/10.1086/426127.

\section{Permanent link}

http://nrs.harvard.edu/urn-3:HUL.InstRepos:41399927

\section{Terms of Use}

This article was downloaded from Harvard University's DASH repository, and is made available under the terms and conditions applicable to Other Posted Material, as set forth at http:// nrs.harvard.edu/urn-3:HUL.InstRepos:dash.current.terms-of-use\#LAA

\section{Share Your Story}

The Harvard community has made this article openly available.

Please share how this access benefits you. Submit a story.

\section{Accessibility}




\title{
The hard quiescent spectrum of the neutron-star X-ray transient EXO 1745-248 in the globular cluster Terzan 5
}

\author{
Rudy Wijnands ${ }^{1,2}$, Craig O. Heinke ${ }^{3}$, David Pooley ${ }^{4}$, Peter D. Edmonds ${ }^{3}$, Walter H. G. \\ Lewin $^{4}$, Jonathan E. Grindlay ${ }^{3}$, Peter G. Jonker ${ }^{6,3}$, Jon M. Miller ${ }^{3}$
}

\begin{abstract}
We present a Chandra observation of the globular cluster Terzan 5 during times when the neutron-star X-ray transient EXO 1745-248 located in this cluster was in its quiescent state. We detected the quiescent system with a $(0.5-10 \mathrm{keV})$ luminosity of $\sim 2 \times 10^{33} \mathrm{ergs} \mathrm{s}^{-1}$. This is similar to several other neutronstar transients observed in their quiescent states. However, the quiescent Xray spectrum of EXO 1745-248 was dominated by a hard power-law component instead of the soft component that usually dominates the quiescent emission of other neutron-star X-ray transients. This soft component could not conclusively be detected in EXO 1745-248 and we conclude that it contributed at most $10 \%$ of the quiescent flux in the energy range $0.5-10 \mathrm{keV}$. EXO $1745-248$ is only the second known neutron-star transient whose quiescent spectrum is dominated by the hard component (SAX J1808.4-3658 is the other one). We discuss possible explanations for this unusual behavior of EXO 1745-248, its relationship to other quiescent neutron-star systems, and the impact of our results on understanding quiescent X-ray binaries. We also discuss the implications of our results on the way the low-luminosity X-ray sources in globular clusters are classified.
\end{abstract}

Subject headings: accretion, accretion disks — stars: individual (EXO 1745248) - X-rays: stars - globular clusters: individual(Terzan 5)

\footnotetext{
${ }^{1}$ School of Physics and Astronomy, University of St Andrews, North Haugh, St Andrews, Fife, KY16 9SS, Scotland, UK

2 Present address: Astronomical Institute "Anton Pannekoek", University of Amsterdam, Kruislaan 403, 1098 SJ, Amsterdam, The Netherlands; rudy@science.uva.nl

${ }^{3}$ Harvard-Smithsonian Center for Astrophysics, 60 Garden Street, Cambridge, MA 02139, USA

${ }^{4}$ Center for Space Research, Massachusetts Institute of Technology, 77 Massachusetts Avenue, Cambridge, MA 02139, USA

${ }^{5}$ Institute of Astronomy, Madingley Road, CB3 0HA, Cambridge, UK
} 


\section{Introduction}

Low-mass X-ray binaries (LMXBs) are binary systems in which a compact object (either a neutron star or a black hole) accretes matter from a low-mass companion star. The neutronstar X-ray transients are a sub-group of LMXBs which spend most of their time in a quiescent state during which hardly any or no accretion onto the neutron star takes place. Occasionally, huge increases in the mass-accretion rates occur making those systems very luminous (with X-ray luminosities up to $\sim 10^{38} \mathrm{ergs}^{-1}$ ). In their quiescent states, neutron-star X-ray transients can still be detected with sensitive X-ray instruments at observed luminosities $(0.5-10 \mathrm{keV})$ of $\sim 10^{32} \mathrm{ergs} \mathrm{s}^{-1}$ to a few times $10^{33} \mathrm{ergs} \mathrm{s}^{-1}$ (see, e.g., van Paradijs et al. 1987; Asai et al. 1996, 1998). Usually, the spectra of those systems in their quiescent states are dominated by a soft component below $1 \mathrm{keV}$ which is thought to be thermal emission from the neutron-star surface due to the cooling of the neutron-star core which has been heated during the outbursts (Campana et al. 1998; Brown, Bildsten \& Rutledge 1998). An additional power-law shaped component which dominates above a few keV might be present in the quiescent spectra. This hard component can contribute up to half of the emission in the 0.5-10 keV energy range (e.g., Asai et al. 1998; Rutledge et al. 2001a). However, this power-law component cannot be detected in all observed quiescent neutron-star systems and the flux ratio of the power-law component to the thermal component varies considerably between sources. The origin of this hard component is not well understood but it might be due to residual accretion down to the magnetospheric radius or it may be a result of the pulsar emission mechanism being active (e.g., Stella et al. 1994; Campana et al. 1998; Campana \& Stella 2000; Menou \& McClintock 2001).

Currently, only one neutron-star X-ray transient does not follow this typical quiescence behavior. Campana et al. (2002) found that the accretion-driven millisecond X-ray pulsar SAX J1808.4-3658 had a very low quiescent luminosity of only $\sim 5 \times 10^{31} \mathrm{ergs} \mathrm{s}^{-1}$ and its quiescent spectrum was dominated by a hard component with no significant detection of the thermal component. They suggested that the very low luminosity of the thermal component might indicate that the neutron star in SAX J1808.4-3658 is rather cool. Such a cold neutron star suggests that enhanced cooling processes are active in its core, possibly because it is a relatively massive neutron star (with a mass of $>1.7 \mathrm{M}_{\odot}$; Colpi et al. 2001). They also suggested that the hard spectral component possibly originates in the shock between the wind of a turned-on radio pulsar and matter outflowing from the companion star (see also Stella et al. 2000). Alternatively, the quiescent X-rays could be due to direct dipole radiation from the radio pulsar (Di Salvo \& Burderi 2003; Burderi et al. 2003). It is still unclear why SAX J1808.4-3658 in its quiescent state is different from the other quiescent neutron-star transients. Its pulsating nature during outburst distinguishes this source from most other neutron-star transients and could be related to its unusual quiescent properties. 
More neutron-star transients have to be observed in quiescence before we can begin to understand SAX J1808.4-3658.

The four other accreting millisecond pulsars (see Wijnands 2003 for a review) are prime candidates and three of them will indeed be observed by either XMM-Newton or Chandra in 2004 (see Wijnands et al. 2004 for the first results). This will clarify whether the unusual quiescent properties of SAX J1808.4-3658 are related to its pulsating nature during outburst. In addition, observing and studying in detail as many non-pulsating neutron-star transients as possible during their quiescent state will lead to a consensus on the quiescent luminosity and spectral-shape distribution of those systems. Here we report on a Chandra observation of the globular cluster Terzan 5 which is known to harbor the neutron-star transient EXO 1745-248. Our observation was taken during times when this transient was in its quiescent state. We found that during its quiescent state the source exhibited a hard X-ray spectrum similar to SAX J1808.4-3658 although its 0.5-10 keV luminosity was a factor of $\sim 40$ higher than that of SAX J1808.4-3658. This is the first time that a neutron-star transient which does not exhibit pulsations during outburst is observed to exhibit the same spectral behavior as the millisecond X-ray pulsar SAX J1808.4-3658 in quiescence.

\section{Observations and analysis}

A previous Chandra observation of the globular cluster Terzan 5 was performed to study the low-luminosity X-ray sources in this cluster, including the identification and study of the quiescent counterpart of EXO 1745-248. However, it was found that the neutron-star transient EXO 1745-248 was in a rare bright state during this observation (Heinke et al. 2003a; see also Fig.1). Although this directly resulted in a sub-arcsecond position for the transient (the errors on the position were $\sim 0.5^{\prime \prime}$; Wijnands, Homan \& Remillard 2002; Heinke et al. 2003a), its very bright wings (because of the point-spread-function of the instrument) did not allow Heinke et al. (2003a) to study the low-luminosity X-ray sources in detail (only nine low-luminosity sources could be detected). To obtain the original goals, we re-observed the cluster using Chandra on 13-14 July 2003 with a total exposure time of $\sim 39.3 \mathrm{ksec}$ (using the ACIS-S3 chip). In Figure 1 we show the Rossi X-ray Timing Explorer (RXTE) All Sky Monitor (ASM) light curve of the source since January 1999 (part of this light curve was already shown by Heinke et al. 2003a). In this figure we indicate when our Chandra observation was made, showing that at this time EXO 1745-248 was in its quiescent state. The Chandra data were reduced and analyzed using CIAO 3.1 and following the thread listed 
on the CIAO web pages ${ }^{1}$. We found that during the final stages of the observation several bright background flares occurred. These flares were excluded from all subsequent analyzes resulting in a total usable exposure time of $\sim 31.2 \mathrm{ksec}$.

\subsection{Image analysis}

We produced an image of the cluster for the data obtained on 13-14 July 2003 (see Fig. 2 right panel) and for comparison we made a similar image for the data obtained during the observation when EXO 1745-248 was in outburst (Fig. 2 left panel; see also Heinke et al. 2003a). The images were created after we had reprocessed the data to remove the pixel randomization added in the standard processing. This reprocessing slightly enhances the spatial resolution of the images. From Figure 2 (right) it can be seen that the transient was indeed observed during its quiescent state. We used the tool 'wavdetect' on the quiescent data to search for low-luminosity point sources and to obtain sub-arcsecond positions ${ }^{2}$ for those sources. Due to the crowding in the image, wavdetect did not detect all sources in the center of the cluster and several sources had to be added by hand. Including those sources, we detected a total of 61 sources within two half-mass radii of the cluster. This number of detected sources is a significant improvement over the ten sources (including the transient) detected by Heinke et al. (2003a). All the nine low-luminosity sources detected by Heinke et al. (2003a) during the outburst observation were again detected during our quiescent observation. To identify the individual sources, we use the source numbering used by Heinke et al. (2003a) in the remainder of this paper. Several of the 61 detected sources are sufficiently bright to expect that they should have been detected during the outburst observation if they had been equally bright during both observations, suggesting that they are highly variable. Moreover, several of the nine sources detected by Heinke et al. (2003a) are also variable between the two observations. The analysis and discussion of those low-luminosity globular cluster sources will be reported in a forthcoming paper (Heinke et al. 2004 in preparation).

By comparing the outburst and quiescent images, the most likely counterpart of the transient in its quiescent state can be identified (see Fig. 2 right; the arrow indicates the likely quiescent counterpart). Using differential astrometry between the outburst and quiescent observations, we can determine whether this likely counterpart candidate is indeed EXO 1745-248 in its quiescent state. To do that, we calculated the coordinate offset of each

\footnotetext{
${ }^{1}$ Available at http://cxc.harvard.edu/ciao/

${ }^{2}$ In general, the errors on the coordinates of the detected sources are dominated by the satellite pointing error which is approximately $0.6^{\prime \prime}$ (90\% confidence levels; see http://cxc.harvard.edu/cal/ASPECT/celmon/).
} 
source $^{3}$ detected by Heinke et al. (2003a) between the outburst and quiescent observations. We found an averaged offset of $0.48^{\prime \prime}$ and $-0.11^{\prime \prime}$ for the right ascension and declination, respectively. The standard deviations on these offsets are $0.13^{\prime \prime}$ for the right ascension and $0.20^{\prime \prime}$ for the declination. The coordinates given for EXO 1745-248 by Heinke et al. (2003a) were offset by $0.48^{\prime \prime}$ in right ascension and $-0.19^{\prime \prime}$ in declination from the (uncorrected) coordinates of the candidate quiescent counterpart (indicated by the arrow in Fig. 2, right panel). These offsets are well within the range of offsets measured for the other sources.

From the above, we see that the two X-ray images can be tied to each other to within $\sim 0.2^{\prime \prime}$ (i.e., the standard deviations on the offsets). We can now estimate the likelihood for a random source to fall within $0.2^{\prime \prime}$ of the source position of EXO 1745-248. We calculated this probability using the source density in one core radius of the globular cluster (which is 7.9"; Cohn et al. 2002). We detected 13 sources in this core radius which gives a probability of $0.8 \%$ that one of those 13 sources fall within $0.2^{\prime \prime}$ of the position of EXO $1745-248$. We note that this probability is conservative since the source density increases toward the cluster center and EXO 1745-248 is located close to the edge of the core where the source density is less. Moreover, the source detected on the position of EXO 1745-248 has unusual properties for a low-luminosity globular cluster X-ray source: it has a relatively high X-ray luminosity and hard X-ray spectrum (see $\S 2.2$ ). Those sources are very unusual in globular clusters and the chances for such a peculiar source (of which there are two within the core radius of Terzan 5; see Fig. 2 and Heinke et al. 2004 in preparation) to fall within $0.2^{\prime \prime}$ of the position of EXO $1745-248$ is $0.12 \%$. Therefore, we conclude that this low-luminosity source is indeed the quiescent counterpart of the transient EXO 1745-248 and that it has peculiar characteristics in its quiescent state (as discussed below).

\subsection{Spectral analysis}

From Figure 2 (right) it can be seen that the quiescent counterpart was not the brightest $\mathrm{X}$-ray source in the cluster and that it had somewhat blueish X-ray colors indicating a reasonably hard spectrum. To quantify this, we extracted the quiescent spectrum of the source. We used the randomized data to extract the spectrum since no calibration products are available for data with the randomization removed. We used a circle with a radius

\footnotetext{
${ }^{3}$ We did not use source eight because it had significantly larger errors on its coordinates than the other sources and this source dominated the standard deviation of the averaged offset. We note that the quiescent data were not tied to any optical/IR image as was done for the outburst image (Heinke et al. 2003a). However, for our purposes we are only interested in differential astrometry between the quiescent and outburst image. The absolute astrometry of the quiescent image will be discussed by Heinke et al. 2004, in preparation.
} 
of $1.5^{\prime \prime}$ centered on the source position as the extraction region. Owing to the crowding near the position of the transient, the standard practice of using an annulus around the source position as extraction region for the background could not be applied. Therefore, as background region we used a circle with a radius of $40^{\prime \prime}$ which was $1.3^{\prime}$ to the west of EXO 1745-248 but which did not contain any point sources. We used the CIAO tool 'psextract' to extract the source and background spectra and to create the response matrix and the ancillary response files (the latter was automatically corrected for the time-variable lowenergy quantum efficiency degradation ${ }^{4}$ ). We grouped the spectrum in bins of 15 counts in order to validate the use of the $\chi^{2}$ fitting method and fitted the spectrum using Xspec (Arnaud 1996; V11.3.1).

The source spectrum is shown in Figure 3 (a total of $\sim 250$ counts were detected from EXO 1745-248; see $\S 2.3$ ). Clearly, the source is significantly detected up to at least 6 $\mathrm{keV}$ confirming the hardness of the spectrum. We fitted the spectrum using a variety of models. We found that a simple power-law model modified by the Galactic absorption column density $N_{\mathrm{H}}$ (using 'phabs' in Xspec; the column density was left as a free parameter in the fits) toward Terzan 5 could adequately fit the data (reduced $\chi^{2}$ of 0.29 with 13 degrees of freedom [d.o.f. $\left.]^{5}\right)$. The obtained $N_{\mathrm{H}}$ was $1.4_{-0.4}^{+0.5} \times 10^{22} \mathrm{~cm}^{-2}$ which is consistent with the infrared-derived estimate for the column density of Cohn et al. (2002; who obtained $\left.1.2 \times 10^{22} \mathrm{~cm}^{-2}\right)^{6}$. The power-law index obtained was $1.8_{-0.4}^{+0.5}$ and the unabsorbed $0.5-10 \mathrm{keV}$ flux was $2.2_{-0.3}^{+0.7} \times 10^{-13} \mathrm{ergs} \mathrm{cm}^{-2} \mathrm{~s}^{-1}$. Normally quiescent neutron-star systems are fit with a thermal model, i.e., a neutron-star atmosphere (NSA) model (Zavlin, Pavlov, \& Shibanov 1996, Gänsick, Braje, \& Romani 2002). In those NSA models, the normalization is given as $1 / d^{2}$ with $d$ the distance in pc. The distance toward Terzan 5 is given as $8.7 \pm 3 \mathrm{kpc}$ (Cohn et al. 2002; Kuulkers et al. 2003), resulting in a $1 / d^{2}$ of $1.32 \times 10^{-8}$, which was used as

\footnotetext{
${ }^{4}$ See http://asc.harvard.edu/cal/Acis/Cal_prods/qeDeg/

${ }^{5}$ The reduced $\chi^{2}$ is rather low and might suggest that the $\chi^{2}$ method cannot be used for our data set. We have checked our results by using different number of counts per spectral bin, by using the Cash statistics (Cash 1979), and by using both. The results obtained with those alternative methods were always consistent with those obtained with the $\chi^{2}$ method using 15 counts per bin (i.e., the fractional uncertainties obtained when calculating errors using Cash statistics are very similar to those measured when using $\chi^{2}$ statistics). Therefore, we only present the results obtained via the latter method.

${ }^{6}$ During outburst, Kuulkers et al. 2003 reported column densities which could be up to three times larger than that measured for the quiescent counterpart of EXO 1745-248. However, during outburst EXO 1745248 can exhibit strong dipping behavior (e.g., Markwardt \& Swank 2000) suggesting that those measured column densities are due to internal absorption in the system. Furthermore, Heinke et al. 2003a reported on a Chandra outburst observation of this source and they measured a column density which is more consistent with the infrared-derived estimate (albeit still slightly larger) also suggesting variable internal absorption.
} 
normalization in our initial fits using the two NSA models. We also assumed a 'canonical' neutron star with a mass of $1.4 \mathrm{M}_{\odot}$ and a radius of $10 \mathrm{~km}$. Therefore, in the NSA models only the temperature was allowed to float as a free parameter. However, when fitting the quiescent spectrum, we found that such models did not provide a good fit with a reduced $\chi^{2}>5.3$ (using 14 d.o.f.). Leaving the neutron-star mass and/or radius free in the fits also did not result in an acceptable description of our spectrum (reduced $\chi^{2}>3.3 ; 12$ d.o.f.) Furthermore, when leaving those parameters free in the fits, they could not be constrained.

Leaving the normalization free and the column density as a free parameter (but again assuming a canonical neutron star) resulted in an acceptable fit for the NSA model from Zavlin et al. (1996) but with an effective temperature $\left(T_{\text {eff }}\right)$ of $\sim 0.6 \mathrm{keV}$ (for an observer at infinity) and a normalization of $\sim 4.2 \times 10^{-12}$. This temperature is a factor of $\sim 3$ larger than usually seen for quiescent neutron-star systems and the normalization implies an extremely large distance toward the source of $\sim 490 \mathrm{kpc}$. Therefore, we conclude that this NSA model does not provide a good description of the data. The NSA model of Gänsick et al. (2002) did not allow for a good fit because the temperature soon reached the maximum allowed temperature in that model $\left(\log T_{\text {eff }}=6.5\right)$ suggesting that also this NSA model does not describe our data. For comparison with results in the literature for other quiescent neutronstar transients, we also fit the data with a black-body model, but the resulting temperature was $1.0 \pm 0.2 \mathrm{keV}$, again significantly larger than seen in any other quiescent neutron-star system (the resulting column density was $0.6 \pm 0.3 \mathrm{~cm}^{-2}$ and the reduced $\chi^{2}$ was 0.36 with 12 d.o.f.). Furthermore, the obtained radius of the emitting region was very small (only $0.10 \pm 0.04 \mathrm{~km}$; assuming a distance of $8.7 \mathrm{kpc}$ ) also suggesting that a black-body model is not a good description of our data.

Several quiescent neutron-star transients have a quiescent spectrum which consists of a soft, thermal component and an additional hard component which dominates above a few $\mathrm{keV}$. One can assume that EXO 1745-248 has a similar composite quiescent spectrum and that the relatively high column density toward Terzan 5 obscures most (if not all) of the thermal component. To determine the flux upper limit on such a soft component we fitted the spectrum using the NSA models plus a power-law component. We again assumed a canonical neutron star and fixed the normalization in the NSA models to $1 / d^{2}=1.32 \times 10^{-8}$. The only free parameter of the NSA models was again the temperature. Such models resulted in a best fit value for $T_{\text {eff }}$ (for an observer at infinity) of $<0.08 \mathrm{keV}$ with an unabsorbed $0.5-10$ $\mathrm{keV}$ flux of $0.1-0.2 \times 10^{-13} \mathrm{ergs} \mathrm{cm}^{-2} \mathrm{~s}^{-1}$ for both the Zavlin et al. (1996) and Gänsick et al. (2002) NSA models (the bolometric flux was approximately twice these values; the power-law index and column density were consistent with that obtained when fitting the data with only a power-law component). These upper limits on the effective temperatures are close to the temperatures observed for other quiescent neutron-star transients, however, the contribution 
of the thermal component to the unabsorbed $0.5-10 \mathrm{keV}$ flux is only $5 \%-7 \%$. Changing the normalization (hence the distance; we used a range between 6 and $12 \mathrm{kpc}$ ) only resulted in a maximum contribution of the thermal component to the unabsorbed $0.5-10 \mathrm{keV}$ flux of $8 \%-10 \%$, strongly suggesting that the quiescent spectrum of EXO 1745-248 is more than $90 \%$ dominated by the hard spectral component.

A different way of demonstrating that the quiescent spectrum of EXO 1745-248 is dominated by the hard component is assuming that both the thermal and hard component contribute $\sim 50 \%$ to the unabsorbed flux in the $0.5-10 \mathrm{keV}$ energy range. We fixed the normalization of the power-law component in such a way that this component always contributed $50 \%$ to the $0.5-10 \mathrm{keV}$ flux. This time we allowed both the temperature of the NSA models and the normalization to be free parameters during the fits (again we assumed a canonical neutron star). This resulted in relatively high effective temperatures ( $T_{\text {eff }}$ for an observer at infinity of $>0.3 \mathrm{keV}$ ) but with unrealistically large distances of up to several Mpc. Therefore, such models do not provide a realistic description of the spectrum, suggesting again that the spectrum is dominated by the hard power-law component with only a small contribution from the thermal component.

Finally, we fitted the spectrum with a thermal bremsstrahlung spectrum which could fit the data satisfactorily (reduced $\chi^{2}$ of 0.24 with 12 d.o.f.). The column density obtained was $1.2 \pm 0.4 \times 10^{22} \mathrm{~cm}^{-2}$, again very similar to the inferred column density toward Terzan 5 , and the plasma temperature was $8_{-4}^{+17} \mathrm{keV}$. The unabsorbed $0.5-10 \mathrm{keV}$ flux was $2.0 \times 10^{-13}$ ergs $\mathrm{cm}^{-2} \mathrm{~s}^{-1}$.

\subsection{Timing analysis}

The number of counts detected for EXO 1745-248 (using the same source and background regions as in the spectral analysis above) was $250 \pm 16$ counts $(0.3-10 \mathrm{keV})$, resulting in a background-corrected count rate of $0.0080 \pm 0.0005$ counts s$^{-1}$ (averaged over the entire observation). To determine the amount of variability in the count rate throughout the observation, we created X-ray count rate curves of the source. In Figure 4 the count rate curve is shown using a time resolution of 2000 seconds. This figure shows that the source was variable during our observation. We applied Kolmogorov-Smirnov and Cramer-Von Mises tests (using the method outlined in Heinke et al. 2003a) on the $0.5-8 \mathrm{keV}$ event list of the source to attempt to disprove the hypothesis that the source flux is constant. Both tests show that EXO 1745-248 is variable at the 95\% confidence level. 


\section{Discussion}

We have presented a Chandra observation of the globular cluster Terzan 5 during times when the neutron-star X-ray transient EXO 1745-248, known to be a member of this cluster, was in its quiescent state. The transient was detected at a $0.5-10 \mathrm{keV}$ luminosity of $\sim 2 \times 10^{33}$ $\operatorname{ergs~s}^{-1}$ for a distance of $8.7 \mathrm{kpc}$ (Cohn et al. 2002; Kuulkers et al. 2003). This quiescent luminosity of EXO 1745-248 is similar to that observed for several quiescent neutron-star systems (e.g., 4U 1608-52; Aql X-1; 4U 2129+47; Asai et al. 1998; Rutledge et al. 2001b; Nowak et al. 2002). However, in contrast to those systems, the quiescent spectrum of EXO 1745-248 is not dominated by the thermal component but by the hard spectral component. We estimate that the soft component contributes at most 5\%-10\% (depending on the exact spectral model used and the uncertainties in the distance) of the flux in the $0.5-10 \mathrm{keV}$ energy range (thus a luminosity upper limit of $1-2 \times 10^{32} \mathrm{ergs} \mathrm{s}^{-1}$ ) and that the temperature of the thermal component is very low $(<0.1 \mathrm{keV})$. The source is also variable at the $95 \%$ confidence level.

\subsection{Thermal emission from the neutron star in EXO 1745-248}

The standard cooling model (which assumes standard core cooling processes; see $\S 1$ ) is commonly used to explain the thermal emission of quiescent neutron-star systems. In this model, the expected quiescent flux $\left(F_{\mathrm{q}}\right)$ of a particular system depends on its long-term timeaveraged (averaged over $>10,000$ years) accretion history and is given by $F_{\mathrm{q}} \approx\langle F\rangle / 135$ (Wijnands et al. 2001; Rutledge et al. 2002) with $\langle F\rangle$ the time averaged flux due to accretion. The latter can be rewritten as $\langle F\rangle=t_{\mathrm{o}}\left\langle F_{\mathrm{o}}\right\rangle /\left(t_{\mathrm{o}}+t_{\mathrm{q}}\right)$ resulting in $F_{\mathrm{q}} \approx \frac{t_{\mathrm{o}}}{t_{\mathrm{o}}+t_{\mathrm{q}}} \times \frac{\left\langle F_{\mathrm{o}}\right\rangle}{135}$, with $\left\langle F_{\mathrm{o}}\right\rangle$ the average flux during outburst, $t_{\mathrm{o}}$ the average time the source is in outburst, and $t_{\mathrm{q}}$ the average time the source is in quiescence. EXO 1745-248 has been detected in outburst on several occasions ${ }^{7}$ : August 1980 (Makishima et al. 1981; type-I X-ray bursts were seen indicating active accretion onto the neutron star but no persistent emission could be found), June 1984 (Warwick et al. 1988), August 1990 (Verbunt et al. 1995), March 1991 (Johnston, Verbunt, \& Hasinger 1995), July-August 2000 (Markwardt \& Swank 2000; Kuulkers et al. 2003), and June 2002 (Wijnands et al. 2002). The observations of the source obtained during all outbursts before the 2000 outburst constitute only a snap-shot of the brightness of the

\footnotetext{
${ }^{7}$ Due to the limited angular resolution of past instruments, it cannot be excluded that another X-ray transient in Terzan 5 was detected during those observations instead of EXO 1745-248. However, only one globular cluster (M15) has so far been found to harbor two bright X-ray binaries (White \& Angelini 2001), making it more likely that the bright transient in Terzan 5 was always EXO 1745-248.
} 
source. It is unknown how long those outbursts lasted and how bright the source became during each of them. The RXTE/ASM light curve (see Fig. 1) is available only for the 2000 and 2002 outbursts and can be used to provide an estimate of the time-averaged accretion flux over the last decade. We calculated the expected quiescent flux of EXO 1745-248 mostly based on what is observed during the 2000 and 2002 outbursts.

During the 2000 and 2002 outbursts, the source was detected for $\sim 109$ and $\sim 30$ days, respectively, with a corresponding time-averaged ASM count rate of $\sim 9.7$ and $\sim 7.9$ counts $\mathrm{s}^{-1}$. No other outbursts were observed during the active lifetime of $R X T E / A S M$ (which has been active for 3076 days; as of July 23, 2004). Therefore, averaged over the two observed outbursts, the source was active for 139 days with a time-averaged ASM count rate during outburst of 9.3 count $\mathrm{s}^{-1}$. To calculate the corresponding time-averaged flux of the source, we used PIMMS $^{8}$ to convert this count rate into a flux estimate assuming a power-law shaped spectrum with a power-law index of 2. During our quiescent observation, we have found an $N_{\mathrm{H}}$ of $\sim 1.3 \times 10^{22} \mathrm{~cm}^{-2}$ toward EXO 1745-248, however when the source was in outburst, the observed $N_{\mathrm{H}}$ could be up to three times larger (Kuulkers et al. 2003; likely due to internal absorption in the system; see footnote 6). Therefore, we assumed a range of column density between 1 and $3 \times 10^{22} \mathrm{~cm}^{-2}$ when using PIMMS. This resulted in a time-averaged flux (0.1$100 \mathrm{keV}$ ) during the outbursts of $1.2-1.5 \times 10^{-8} \mathrm{ergs} \mathrm{cm}^{-2} \mathrm{~s}^{-1}$. During the active life-time of the RXTE/ASM the source was active for 139 days and quiescent for 2937 days (as of July $23,2004)$ resulting in a time-averaged flux for the life-time of the ASM of $5.4-6.8 \times 10^{-10}$ $\operatorname{ergs~} \mathrm{cm}^{-2} \mathrm{~s}^{-1}$. Since the source has likely spent more time in quiescence before the launch of $R X T E$, these fluxes should be considered upper-limits on the true time-averaged accretion flux. The outburst history before $R X T E$ is not well known but to first approximation we can assume that no outbursts occurred between the detection of the source in March 1991 (Johnston et al. 1995) and the 2000 outbursts. If true, then the time-averaged source flux is $3.4-4.3 \times 10^{-10} \mathrm{ergs} \mathrm{cm}^{-2} \mathrm{~s}^{-1}$ for the time period between the 1991 detection of the source and the present-day. Because one or more outbursts might have been missed, these flux estimates should be regarded as lower-limits. Combining the two limits, the time-averaged flux of the source due to accretion is likely in the range 3.4 to $6.8 \times 10^{-10} \mathrm{ergs} \mathrm{cm}^{-2} \mathrm{~s}^{-1}$, resulting in a predicted quiescent thermal flux of $2.5-5.0 \times 10^{-12} \mathrm{ergs} \mathrm{cm}^{-2} \mathrm{~s}^{-1}$ (if only standard core cooling is taken into account). However, these predicted values are a factor of $\sim 100$ larger than the flux upper limit we determined for the thermal component in the quiescent data of EXO 1745-248, suggesting that the neutron star is colder than expected, likely owing to the presence of enhanced core cooling processes.

\footnotetext{
${ }^{8}$ Available at http://heasarc.gsfc.nasa.gov/Tools/w3pimms.html
} 
We note that this calculation of the predicted thermal quiescent flux is based on very limited information about the accretion history of the source. Furthermore, we have assumed a simple power-law shaped spectrum of the source during outburst but it has already been shown that this is an oversimplification of the true situation (see, e.g., Kuulkers et al. 2003; Heinke et al. 2003a), resulting in additional uncertainties in the predicted quiescent flux. However, in order for the standard cooling model to agree with our observations, the predicted quiescent flux must drop by a factor of 100, meaning that the quiescent episodes between very bright outbursts (like the 2000 and 2002 ones) should be over a thousand years. This seems unlikely since the source has been detected on several occasions before the two latest outbursts. We prefer the point of view in which the low thermal emission from the neutron star is explained by assuming that enhanced cooling processes occur in the core, especially since strong evidence has been obtained for such processes in the neutron stars of several other quiescent systems (e.g., Brown et al. 1998; Wijnands et al. 2001, 2003; Nowak et al. 2002; Campana et al. 2002). Colpi et al. (2001) proposed that the presence of enhanced core cooling processes indicates that the neutron star should be relatively massive $\left(>1.7 \mathrm{M}_{\odot}\right)$. We note that this conclusion remains valid even if the low-luminosity source we have detected at the position of EXO 1745-248 is not the quiescent counterpart of this transient (although as explained above this is unlikely) but in fact an unrelated source (also likely a member of the globular cluster).

\subsection{The hard spectral component of EXO 1745-248}

The fact that EXO 1745-248 might harbor a cold neutron star may explain why the quiescent spectrum of EXO 1745-248 is dominated by the hard spectral component. Several other systems have exhibited hard components in quiescence which contributed up to $40 \%$ to the $0.5-10 \mathrm{keV}$ quiescent flux ( $(1)$. Wijnands et al. (2003) suggested that if a system was found which has a similar luminous hard component as seen, e.g., for Aql X-1 in its quiescent state (e.g., Asai et al. 1998), but with a neutron star which only emitted very faint thermal emission (e.g., because of its coldness), then the quiescent spectrum of this system should be dominated by the hard spectral component. It seems that EXO 1745-248 might indeed be such a system. We note that this argument assumes that the thermal and hard components are not related to each other and can therefore vary in strength independently. It also assumes that the hard component in EXO 1745-248 is due to the same process which causes the hard components observed in other systems. It is unclear how valid those assumptions

are, mainly because of the lack of understanding of the hard X-ray emission seen in quiescent neutron-star X-ray transients. 
It has been suggested that this hard component might be due to residual accretion onto the surface or magnetic field of the neutron star or to the pulsar mechanism being active (see the discussion in Campana \& Stella 2000). Only limited investigations have been performed to determine exactly how those mechanisms could produce the hard emission, what exactly the spectral shape of the emission would be, and which source properties would make sources differ from each other with respect to this hard spectral component. Currently only two neutron-star systems are known whose quiescent spectra are dominated by the hard spectral component: EXO 1745-248 and SAX J1808.4-3658. Campana et al. (2002) found that SAX J1808.4-3658 is dominated by the hard component in quiescence, although the luminosity of SAX J1808.4-3658 was a factor of 40 lower than that of EXO 1745-246. Again it is unclear whether or not the hard components in these sources are due to the same phenomenon. However, despite this uncertainty, it is clear that hard quiescent spectra can occur at relatively high and very low luminosities and presumably also for luminosities in-between those observed for EXO 1745-248 and SAX J1808.4-3658. Our results also demonstrate that it is not necessary for a source to be an accretion-driven millisecond X-ray pulsar during outburst for its quiescent spectrum to be dominated by the hard component. For EXO 1745-248 no pulsations were seen during the outbursts indicating a different magnetic field structure (e.g., strength, configuration, multi-pole moments) for the neutron star in this system than for the neutron star in SAX J1808.4-3658. However, several models for the hard spectral component involve a combination of the magnetic field strength and configuration, and residual accretion from the companion star, all unknown properties. This gives a large range of source properties one can vary to obtain similar quiescent spectral shapes but different outburst behavior. Insight into the physical process(es) behind the hard spectral component might come from the variability in this component observed in EXO 1745-248 (see Fig. 4).

The hard quiescent spectra of EXO 1745-248 and SAX J1808.4-3658 (Campana et al. 2002) are very similar to the hard quiescent spectra observed for the quiescent black-hole X-ray transients (see Kong et al. 2002 and references therein). It is not clear if the hard spectral component is caused by the same physical process(es) in the quiescent neutron-star transients as in the quiescent black-hole systems, but if they are then any models involving a neutron-star surface or magnetic field or a black-hole event horizon are not valid. Irrespective of whether or not the same physical mechanism(s) is behind the hard quiescent spectra of EXO 1745-248 and SAX J1808.4-3658 and the black-hole systems, our results and those of Campana et al. (2002) demonstrate that not all the neutron-star systems are different in quiescence from the black-hole systems. Rutledge et al. (2000) suggested that these differences could be used to determine the nature of the compact object in an X-ray binary for which this was not yet known. However, our results and those of Campana et al. (2002) 
now show that a hard spectral shape in quiescence does not mean that the compact object in a particular X-ray transient is a black hole (note that on average, the neutron-star systems could still be different from the black-hole systems, but one particular neutron-star binary might not be). On the other hand, the faintness of several of the black-hole systems might still be a property potentially exclusive to those systems and might be used to determine the black-hole nature of certain transients. Unfortunately, several quiescent black-hole systems have quiescent luminosities in the range seen for SAX J1808.4-3658 and EXO 1745-248, making this possible method of distinguishing between the different types of transients only useful for those black-hole systems which can become very faint in quiescence. If the quiescent spectrum of a particular transient is dominated by a soft thermal component, it is still likely that this system harbors a neutron-star primary since no black-hole system has shown a thermal component in its quiescent X-ray spectrum.

Based on the outburst spectrum of EXO 1745-248, Heinke et al. (2003a) suggested that EXO 1745-248 might be in an ultra-compact binary with a binary period $<1$ hour. The 2-hr binary period of SAX J1808.4-3658 (Chakrabarty \& Morgan 1998) shows that this system is in a compact binary system. If the compact binary nature of EXO 1745-248 can be confirmed, this would tentatively indicate that the compact binary nature of this source and SAX J1808.4-3658 might be related to their hard spectra in their quiescent states. However, currently not enough information is available to arrive at any definite conclusions. More information will be available in the next few years after more (ultra-)compact neutron-star binaries have been observed and studied in quiescence (such as XTE J1751-305, XTE J0929314, and XTE J1807-294 which are currently scheduled to be observed with Chandra and/or XMM-Newton; see Wijnands et al. 2004 for the first results).

\subsection{Implication for the classification of low-luminosity globular cluster sources}

The fact that EXO 1745-248 and SAX J1808.4-3658 have hard quiescent spectra may have consequences for our understanding of the nature of the low-luminosity globular cluster $\mathrm{X}$-ray sources. When optical and radio identifications are not available for those sources, they are often given tentative classifications on the basis of their X-ray luminosities and the hardness of their spectra (e.g., Grindlay et al. 2001a, b; Pooley et al. 2002a, b; Gendre, Barret, \& Web 2003; Heinke et al. 2003a). Those sources which have luminosities above $10^{32} \mathrm{ergs} \mathrm{s}^{-1}$ and are soft are generally classified as quiescent neutron-stars systems; those which are relatively bright (up to around $10^{33} \mathrm{ergs} \mathrm{s}^{-1}$ ) and have hard spectra are generally classified as cataclysmic variables $(\mathrm{CVs})$. The sources below $\sim 10^{31} \mathrm{ergs} \mathrm{s}^{-1}$ could be a variety of objects, including CVs, millisecond radio pulsars or active binaries (e.g., BY Dra 
or RS CVn systems). Usually a X-ray color-magnitude diagram is created and different branches are identified corresponding to different source types. To investigate the impact of the hard spectrum of EXO 1745-248 on such a diagram we create a similar diagram for Terzan 5 (Fig. 5). Clearly, the quiescent transient (labeled 'Transient') is one of the brightest sources and its color is quite hard. The sources which are identified by the numbers $2,3,4$, and 8 (using the numbering of Heinke et al. 2003a) are considerably softer and would likely be classified as quiescent neutron-star systems if no additional information were available. Indeed Heinke et al. (2003a) classified them as such and they suggested that the sources to the left of them were good CV candidates. Without prior knowledge of the nature of EXO 1745-248, its hard color would have suggested it to be a CV, but this is far from a clear-cut classification. Moreover, when candidate CVs have enough counts to extract their X-ray spectra, those spectra are usually fit with a thermal bremsstrahlung model with a plasma temperature of $\gtrsim 5 \mathrm{keV}$. As shown in $\S 2.2$, the quiescent spectrum of EXO 1745-248 could also be adequately fitted with such a spectral model and the resulting plasma temperature is fully consistent with what has been observed from CVs. The only unusual aspect of EXO 1745-248 would be its rather high X-ray luminosity of $\sim 2 \times 10^{33} \mathrm{ergs} \mathrm{s}^{-1}$, which would not be fully consistent with a CV nature. Likely, the source would then have been classified as an unusually bright CV, but a potential quiescent neutron-star transient with a very unusual spectrum might also have been suggested.

This possible misclassification strongly suggests that some of the hard sources in the globular cluster studies might have been misclassified as CVs but are in reality quiescent neutron-star systems. This conclusion is further strengthened by the quiescent properties of SAX J1808.4-3658 (Campana et al. 2002). This source had a similar hard quiescent spectrum but a $\sim 40$ times lower X-ray luminosity bringing this source right in the range of luminosity expected for CVs. It is very likely that if SAX J1808.4-3658 had been located in a globular cluster and the neutron-star nature of its primary was not known from additional information (like its outbursts and pulsations), then this source would have been classified on the basis of its quiescent X-ray properties as a CV (see also the discussion in Wijnands et al. 2003). We see no reason why future discovered quiescent neutron-star systems would not exhibit similarly hard spectral shapes and have X-ray luminosities in-between what we observe for EXO 1745-248 and SAX J1808.4-3658. If true, then this group of sources would have very similar X-ray characteristics to those of CVs and this must be taken into account during the classification of low-luminosity X-ray sources in globular clusters when this is done solely on the basis of their X-ray properties.

We searched the literature and found eleven detections of quiescent neutron-star systems for which spectra of sufficient quality could be obtained to investigate their quiescent spectra. We only discuss those systems which are confirmed neutron-star X-ray transients as they 
have been seen to exhibit type-I X-ray bursts and/or X-ray pulsations. Out of those eleven sources, nine were dominated by the thermal component and two were dominated by the hard component $^{9}$. This suggests that out of every eleven quiescent neutron-star systems two such systems could have hard quiescent spectra, which is a non-negligible fraction of the total. Therefore, a similar fraction of hard quiescent neutron-star transients might be present in globular clusters which are currently missed because they are incorrectly classified. We note that this is a very rough estimate mainly because of the very few systems known (especially those with a hard quiescent spectrum). This estimate assumes that most accreting neutronstar systems are similar to those that have been detected in outburst. If the spectral shape in quiescence is related to recent outburst activity, the fraction of hard quiescent neutron-star transients may be larger or smaller than our calculation suggests. Heinke et al. (2003b) compiled a list of 21 quiescent neutron-star systems in globular clusters, identified by their agreement with an NSA model plus an optional power-law component making up $<40 \%$ of the $0.5-10 \mathrm{keV}$ flux. Their results suggest that the strength of a power-law component is correlated with recent outburst activity. However, since their method of identifying quiescent neutron-star systems would not detect all known systems, that correlation cannot yet be considered secure. Reliable optical identifications of numerous hard X-ray sources with cataclysmic variables in 47 Tuc, NGC 6397, and NGC 6752 (Edmonds et al. 2003; Grindlay et al. 2001b; Pooley et al. 2002a) indicate that quiescent neutron-star systems with hard spectra probably do not dominate the quiescent neutron star population in globular clusters.

We used results provided by the RXTE/ASM teams at MIT and at the RXTE SOF and GOF at NASA's GSFC. We especially thank Ron Remillard for reprocessing the $R X T E /$ ASM data to obtain the complete ASM light curve for EXO 1745-248. This research has made use of NASA's Astrophysics Data System and the tools provided by the High Energy Astrophysics Science Archive Research Center (HEASARC), provided by NASA's Goddard Space Flight Center.

\section{REFERENCES}

Arnaud, K. 1996, in G. Jacoby \& J. Barnes (eds.), Astronomical Data Analysis Software and Systems V., Vol. 101, p. 17, ASP Conf. Series.

Asai, K., Dotani, T., Kunieda, H., Kawai, N. 1996, PASJ, 48, L27

\footnotetext{
${ }^{9}$ The soft-component dominated systems are Aql X-1, Cen X-4, 4U 1608-52, 4U 2129+47, KS 1731260, MXB 1659-29, SAX J1748.9-2021 in the globular cluster NGC 6440, RX J170930.2-263927, and SAX J1810.8-2609; the hard-component dominated systems are SAX J1808.4-3658 and EXO 1745-248
} 
Asai, K., Dotani, T., Hoshi, R., Tanaka, Y., Robinson, C. R., Terada, K. 1998, PASJ, 50, 611

Brown, E. F., Bildsten, L., \& Rutledge, R. E. 1998, ApJ, 504, L95

Burderi, L., Di Salvo, T., D’Antona, F., Robba, N. R., Testa, V. 2003, A\&A, 404, L43

Campana, S. \& Stella, L. 2000, ApJ, 541, 849

Campana, S., Colpi, M., Mereghetti, S., Stella, L., Tavani, M. 1998, A\&A Rev., 8, 279

Campana, S., Stella, L., Gastaldello, F., Mereghetti, S., Colpi, M., Israel, G. L., Burderi, L., Di Salvo, T., Robba, R. N. 2002, ApJ, Letters, 575, L15

Cash, W. 1979, ApJ, 228, 939

Chakrabarty, D. \& Morgan, E. H. 1998, Nature, 394, 346

Cohn, H. N., Lugger, P. M., Grindlay, J. E., Edmonds, P. D. 2002, ApJ, 571, 818

Colpi, M., Geppert, U., Page, D., Possenti, A. 2001, ApJ, 548, L175

Di Salvo, T. \& Burderi, L. 2003, A\&A, 397, 723

Edmonds, P. E., Gilliland, R. L., Heinke, C. O., Grindlay, J. E. 2003, ApJ, 596, 1197

Gänsicke, B. T., Braje, T. M., \& Romani, R. W. 2002, A\&A, 386, 1001

Gendre, B., Barret, D., Webb, N. 2003, A\&A, 304, L11

Grindlay, J. E., Heinke, C., Edmonds, P. D., Murray, S.S. 2001a, Science, 292, 2290

Grindlay, J. E., Heinke, C. O., Edmonds, P. D., Murray, S. S., \& Cool, A. M. 2001b, ApJ, $563, \mathrm{~L} 53$

Heinke, C. O., Edmonds, P. D., Grindlay, J. E., Lloyd, D. A., Cohn, H. N., Lugger, P. M. 2003a, ApJ, 590, 809

Heinke, C. O., Grindlay, J. E., Lugger, P. M., Cohn, H. N., Edmonds, P. D., Lloyd, D. A., Cool, A. M., 2003b, ApJ, 598, 501

Johnston, H. M., Verbunt, F., \& Hasinger, G. 1995 A\&A, 298, L21

Kong, A. K. H., McClintock, J. E., Garcia, M. R., Murray, S. S., Barret, D. 2002, ApJ, 570, 277

Kuulkers, E., den Hartog, P. R., in 't Zand, J. J. M., Verbunt, F. W. H. M., Harris, W. E., Cocchi, M. 2003, A\&A, 399, 633

Makishima, K. et al. 1981, ApJ, 247, L23

Markwardt, C. B. \& Swank, J. H. 2000, IAU Circ., 7454

Menou, K. \& McClintock, J. E. 2001, ApJ, 557, 304 
Nowak, M. A., Heinz, S., Begelman, M. C. 2002, ApJ, 573, 778

Pooley, D. et al. 2002a, ApJ, 569, 405

Pooley, D. et al. 2002b, ApJ, 573, 184

Rutledge, R. E., Bildsten, L., Brown, E. F., Pavlov, G. G., Zavlin, V. E. 2000, ApJ, 529, 985

Rutledge, R. E., Bildsten, L., Brown, E. F., Pavlov, G. G., Zavlin, V. E. 2001a, ApJ, 551, 921

Rutledge, R. E., Bildsten, L., Brown, E. F., Pavlov, G. G., Zavlin, V. E. 2001b, ApJ, 559, 1054

Rutledge, R. E., Bildsten, L., Brown, E. F., Pavlov, G. G., Zavlin, V. E., Ushomirsky, G., 2002, ApJ, 580, 413

Stella, L., Campana, S., Colpi, M., Mereghetti, S., Tavani, M., 1994, ApJ, 423, L47

Stella, L., Campana, S., Merghetti, S., Ricci, D., Israel, G. L. 2000, ApJ, 537, L115

van Paradijs, J., Verbunt, F., Shafer, R. A., \& Arnaud, K. A. 1987, A\&A, 182, 47

Verbunt, F., Bunk, W., Hasinger, G., Johnston, H. M. 1995, A\&A, 300, 732

Warwick, R. S., Norton, A. J., Turner, M. J. L., Watson, M. G., Willingale, R. 1988, MNRAS, 232,551

White, N. E. \& Angelini, L. 2001, ApJ, 561, L101

Wijnands, R. 2003 in "The Restless High-Energy Universe", 5-8 May 2003, Amsterdam, The Netherlands, ed. E. P. J. van den Heuvel, J. J. M. in 't Zand, \& R. A. M. J. Wijers (Elsevier) (astro-ph/0309347; the most up-to-date version is available at http://zon.wins.uva.nl/ rudy/admxp/)

Wijnands, R., Miller, J. M., Markwardt, C., Lewin, W. H. G., van der Klis, M. 2001, ApJ, 560, L159

Wijnands, R., Homan, J., \& Remillard, R. 2002, ATEL 101.

Wijnands, R., Nowak, M., Miller, J. M., Homan, J., Wachter, S., Lewin, W. H. G. 2003, ApJ, 594, 952

Wijnands, R., Homan, J., Heinke, C. O., Miller, J. M., \& Lewin, W. H. G., 2004, ApJ, submitted

Zavlin, V. E., Pavlov, G. G., \& Shibanov, Yu. A., 1996, A\&A, 315, 141 


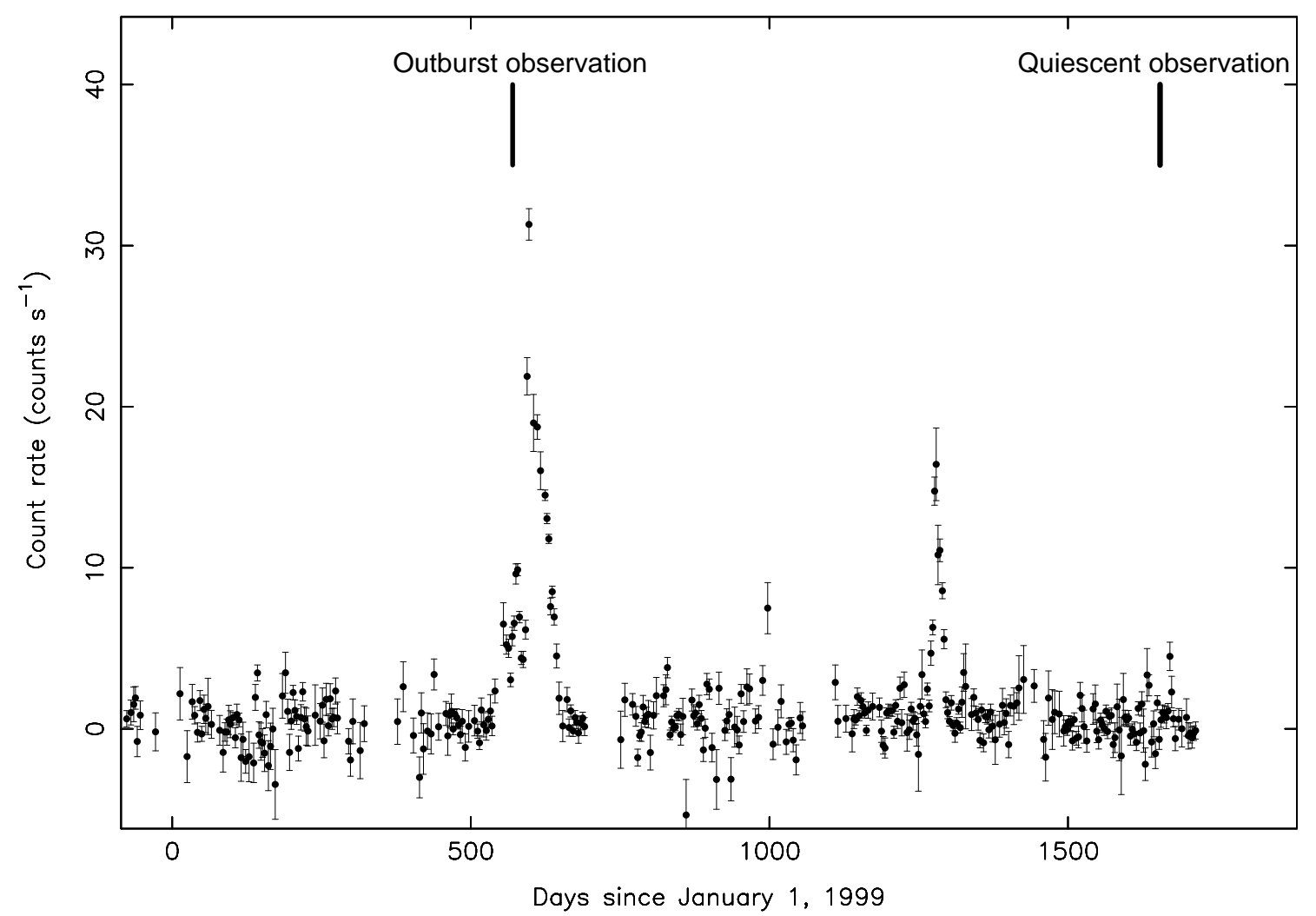

Fig. 1.- The RXTE/ASM light curve of EXO 1745-248. Each point is averaged over three days. The dates of the Chandra observations are marked. 

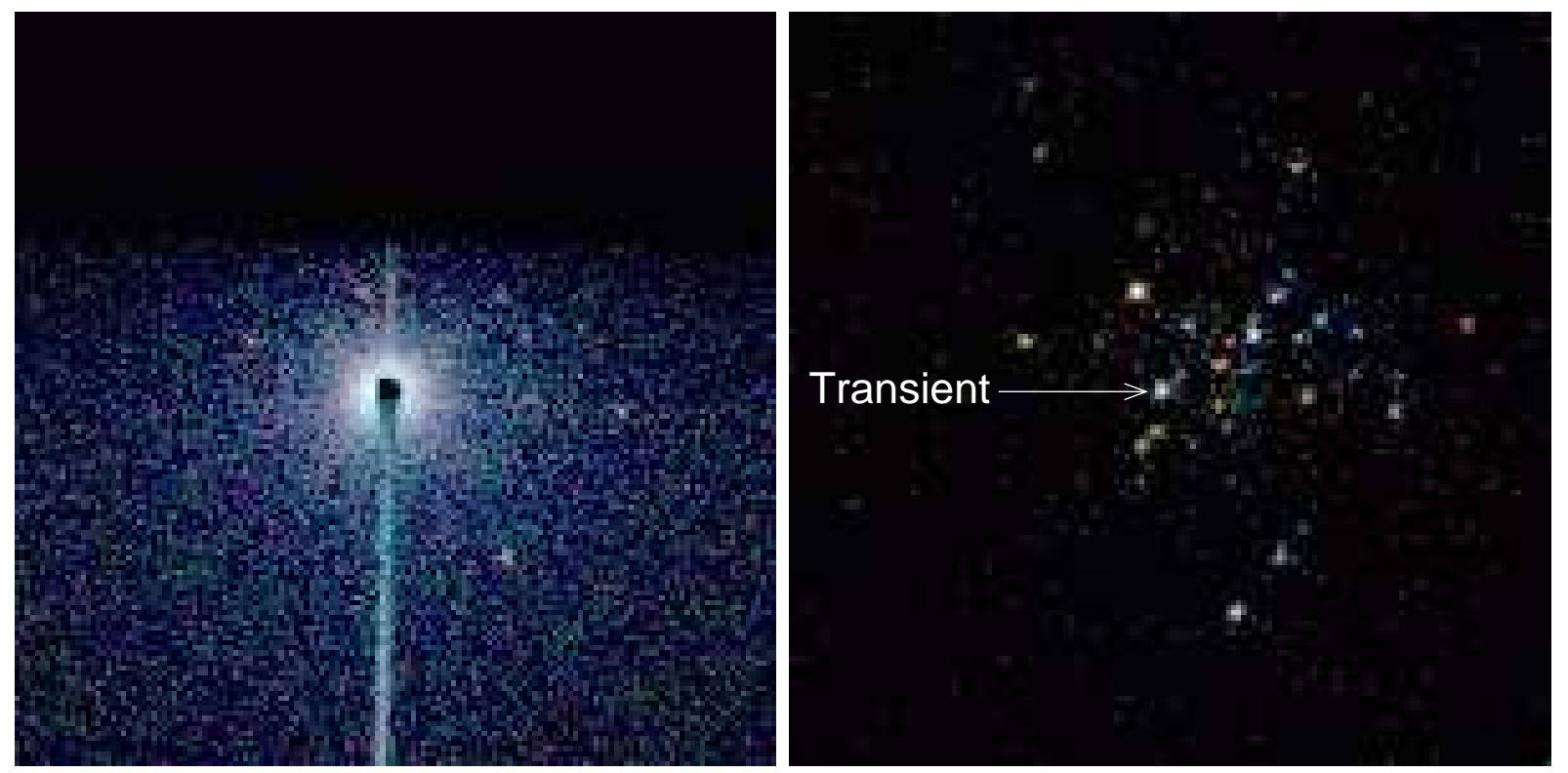

Fig. 2.- The images of the globular cluster Terzan 5. The left panel shows the data obtained in 24 July 2000 during which the transient EXO 1745-248 was very bright (the streak is due to out-of-time events recorded during the frame transfer); the right panel shows the data obtained on 13-14 July 2003 when the transient was in its quiescent state. Both images are plotted on the same scale allowing for direct comparison and are $73.8^{\prime \prime} \times 73.8^{\prime \prime}$. East is toward the left and north is upward. The red color is for the $0.3-1.5 \mathrm{keV}$ energy range, green for $1.5-2.5 \mathrm{keV}$, and blue for $2.5-8.0 \mathrm{keV}$. In the right panel the quiescent transient is indicated by the arrow. The closest source to the quiescent transient is approximately $2.4^{\prime \prime}$ located toward the north-west of the source. 


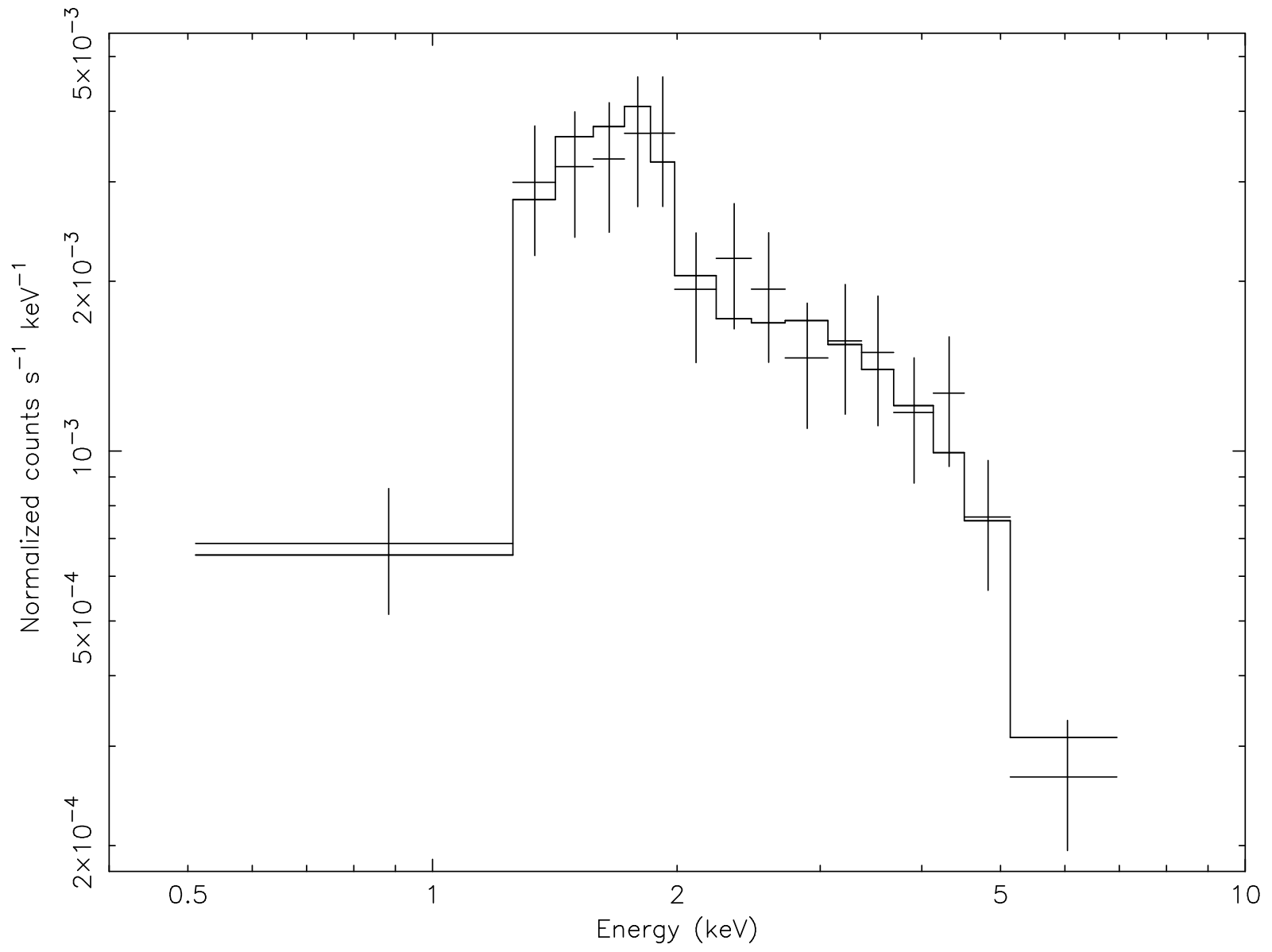

Fig. 3.- The quiescent X-ray spectrum obtained for EXO 1745-248 during the 13-14 July 2003 Chandra observation. The solid line indicates the best fit power-law model. 


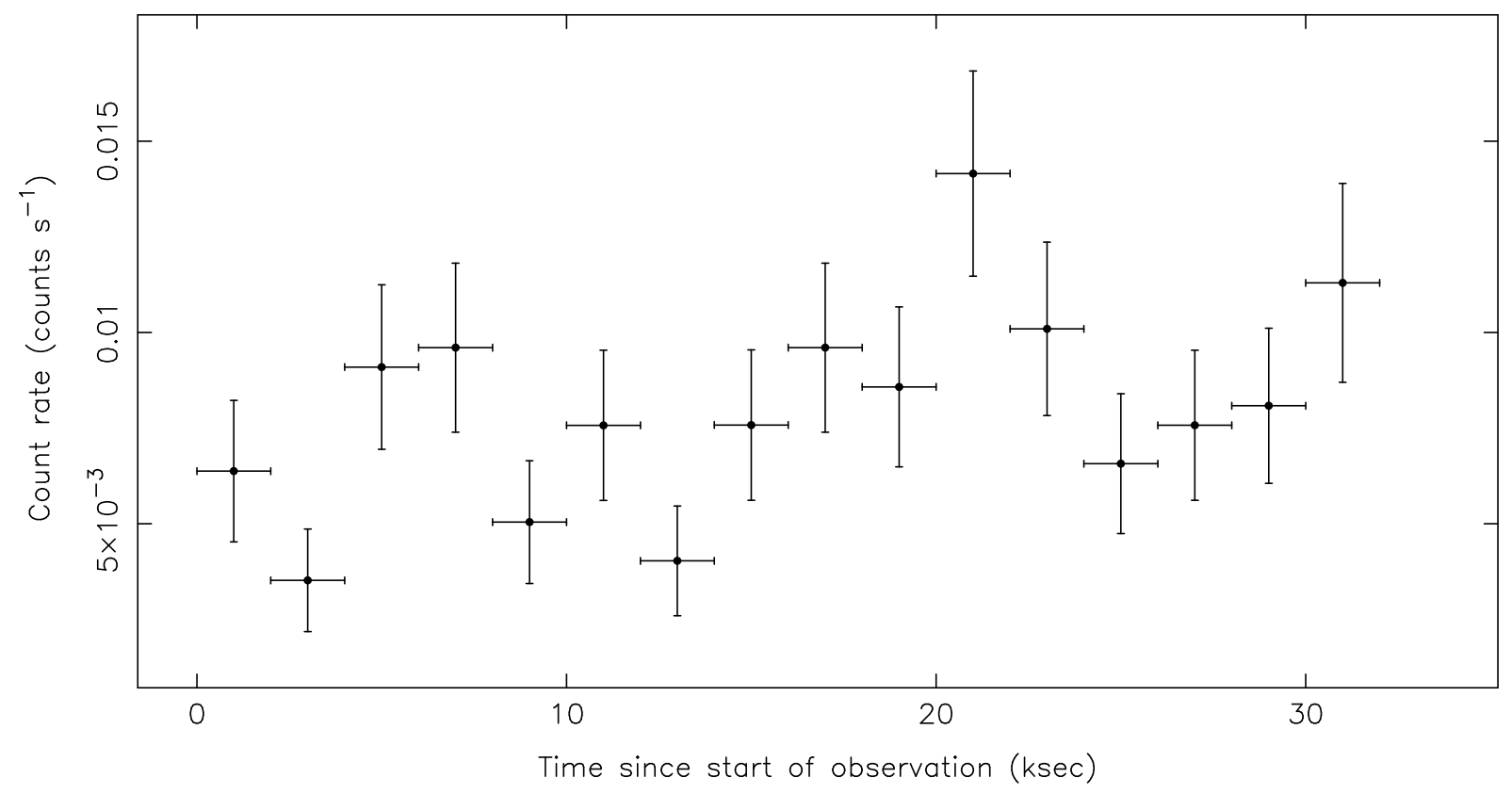

Fig. 4. - The X-ray count rate curve (0.3-10 keV) for EXO 1745-248 during the 13-14 July 2003 Chandra observation. The time resolution is 2000 seconds. 


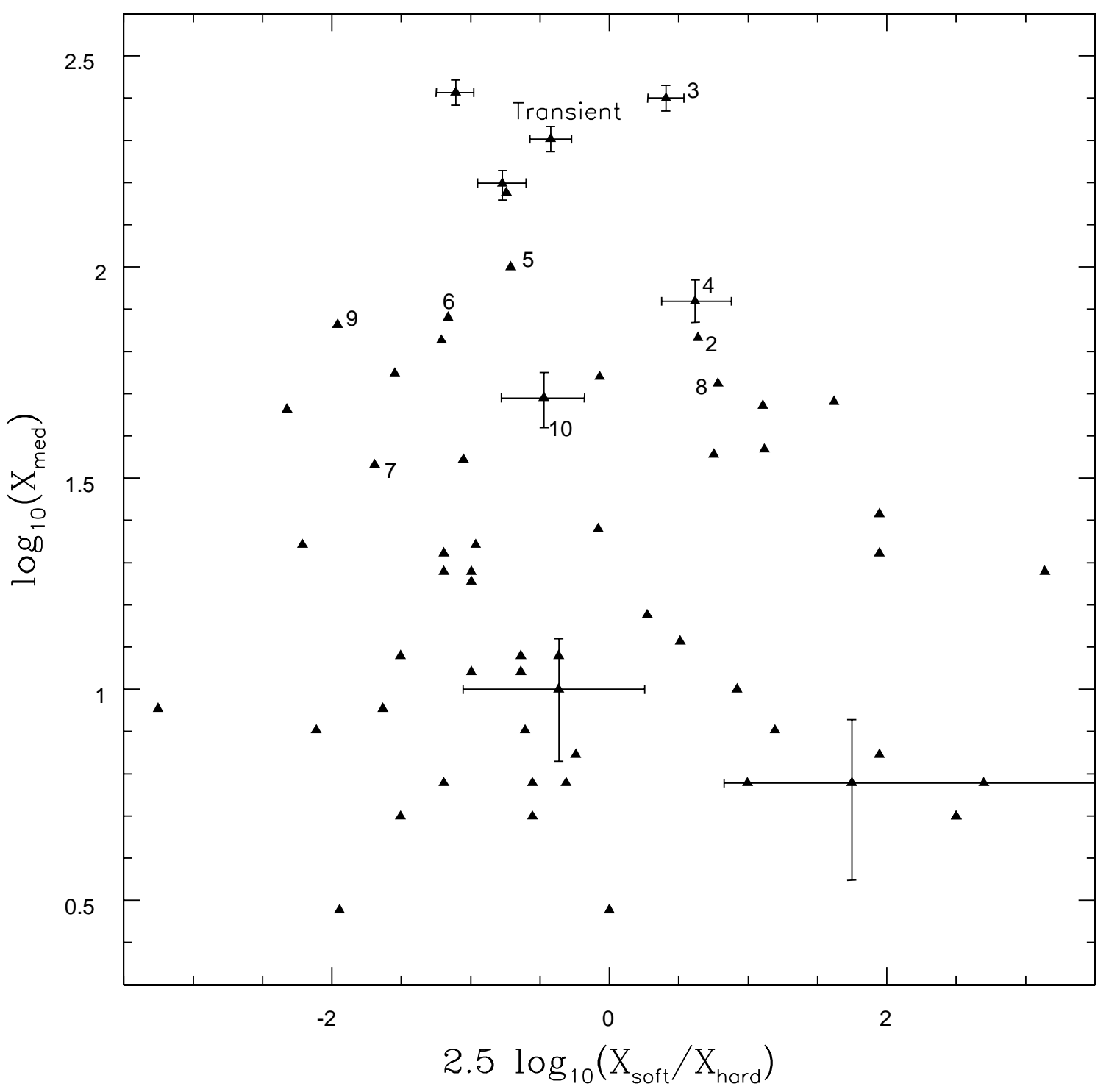

Fig. 5.- The color-magnitude diagram of Terzan 5. The color is defined as the $2.5 \log _{10}$ of the ratio of the number of soft photons $\mathrm{X}_{\text {soft }}$ (for the energy range $0.5-2 \mathrm{keV}$ ) to the number of hard photons $\mathrm{X}_{\text {hard }}$ (for the energy range $2-6 \mathrm{keV}$ ). The magnitude is defined as the $\log _{10}$ of the number of medium hard photons $\mathrm{X}_{\text {med }}$ (for the energy range $0.5-4.5 \mathrm{keV}$ ). In the figure the quiescent neutron-star transient EXO 1745-248 is indicated by the word 'Transient'. The numbers near some of the points correspond to the low-luminosity sources already detected by Heinke et al. (2003) and follows their numbering. For clarity only a few representative error bars are plotted. 